\title{
A Trapped-Ion Phonon Laser
}

\author{
K Vahala*1, M Herrmann, S Knünz, V Batteiger, \\ G Saathoff, T W Hänsch, Th Udem ${ }^{2}$ \\ Max Planck Institute für Quantenoptik, 85748 Garching, Germany \\ *permanent address: California Institute of Technology, Pasadena, California 91125 \\ 'vahala@caltech.edu, ${ }^{2}$ thu@mpq.mpg.de
}

\begin{abstract}
A cooled, trapped $\mathrm{Mg}^{+}$ion is excited using a continuous, blue-detuned pump. Coherent oscillatory motion is observed. It is shown to result from stimulated emission of center-of-mass phonons, constituting the mechanical analog of a laser.
\end{abstract}

(C)2009 Optical Society of America

OCIS codes: $020.3320,140.3320,140.3460$

Red-detuned laser pumping of an atomic resonance will cool the center-of-mass motion of an ion or atom $[1,2]$. This method of cooling has helped make possible a wide range of remarkable scientific discoveries, including realizations for control of quantum information [3,4], production of exotic quantum states $[5,6]$, and unprecedented leaps in metrology [5]. The complementary regime of blue-detuned pumping is investigated in this work. Widely known as the regime of heating, it is instead shown that stimulated emission of center-of-mass phonons occurs here, leading to coherent vibrational motion in analogy with a laser. Both a theory and experimental observations are presented using a single, cooled magnesium ion. The ability to cool the motion (either directly or sympathetically) is significant since thermal quanta can be avoided. The production of semi-classical, vibrational coherence can be a new tool for study of quantum phenomena using ionic systems, or to drive modes within an effective ionic medium.
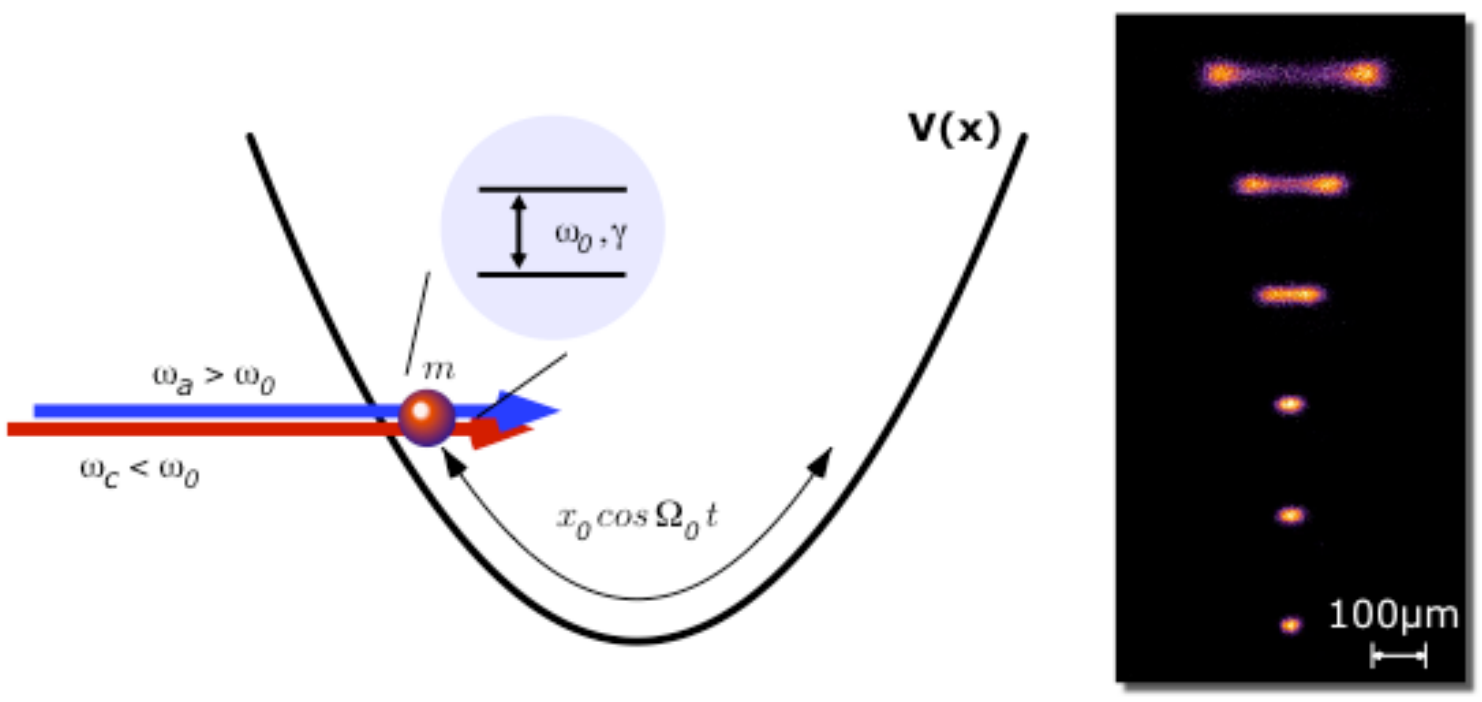

Figure 1: Left panel: ion in a harmonic trap with secular frequency $\Omega_{0}$ excited with both a cooling beam at frequency $\omega_{\mathrm{c}}$ and an amplification beam at frequency $\omega_{\mathrm{a}}$ The ion transition (frequency $\omega_{\mathrm{o}}$ ) has linewidth $\gamma$. Right panel: a series of images of ion luminescence in which the amplification beam intensity is increased from lower to upper images. The cooling beam intensity is constant and the lowest image features no amplification. The ion's lateral motion is time averaged, and a threshold is apparent between the third and fourth images.

A single, magnesium ion in a linear radio frequency trap was studied (figure 1 left panel). Further details on the setup are described in reference [7]. The magnesium D2 transition at $279.6 \mathrm{~nm}$ has a transition linewidth of $41.8 \mathrm{MHz}$ while the axial secular frequency of the trap could be controlled so that the system falls well within the sideband unresolved regime. The axial secular frequency $\left(\Omega_{\mathrm{o}} / 2 \pi\right)$ was set to $71 \mathrm{kHz}$ in all measurements; the micro-motion was negligible. The blue-detuned, amplification beam was applied along the long axis of the ion trap so as to excite only axial oscillations. The red-detuned, cooling 


\section{IFB4.pdf}

beam, on the other hand, was applied slightly off axis from the long axis so as to project onto all three axes of motion. Cooling of the center-of-mass motion to approximately $1 \mathrm{mK}$ was obtained. Imaging the ion motion was possible by monitoring luminescence caused by both the cooling and amplification beams. Figure 1 (right panel) shows a series of such images for increasing levels of amplification beam intensity. The first image shows the ion with no amplification (i.e., only cooling). With increasing amplification beam intensity there is a transition to a double-lobed pattern, produced by time averaging of the oscillatory motion of the ion. Further increases in intensity lead to larger amplitudes. The double-lobed pattern cannot be explained on the basis of heating. Finally, figure 2 shows the squared-amplitude of motion (measured using such images) plotted versus the amplification beam intensity, $i_{a}$ (normalized by the constant, coolingbeam intensity, $i_{c}$ ). The amplification (cooling) beam detuning is set to $+12 \mathrm{MHz}(-74 \mathrm{MHz}$ ). Relative amplification and cooling beam intensities were determined by measuring separately the ion luminescence induced by each beam at a reference, negative detuning. The ion velocity amplitude $\left(\mathrm{v}_{\mathrm{o}}=\Omega_{\mathrm{o}} \mathrm{x}_{\mathrm{o}}\right)$ was determined through traces like that in figure 1 (upper right panel) to first infer the spatial amplitude $x_{0}$. The oscillation threshold is clearly visible in the data. The solid curve in figure 2 is the theoretical operating point curve (there are no free parameters). A theory of phonon stimulated emission and center-of-motion amplification will be reviewed.

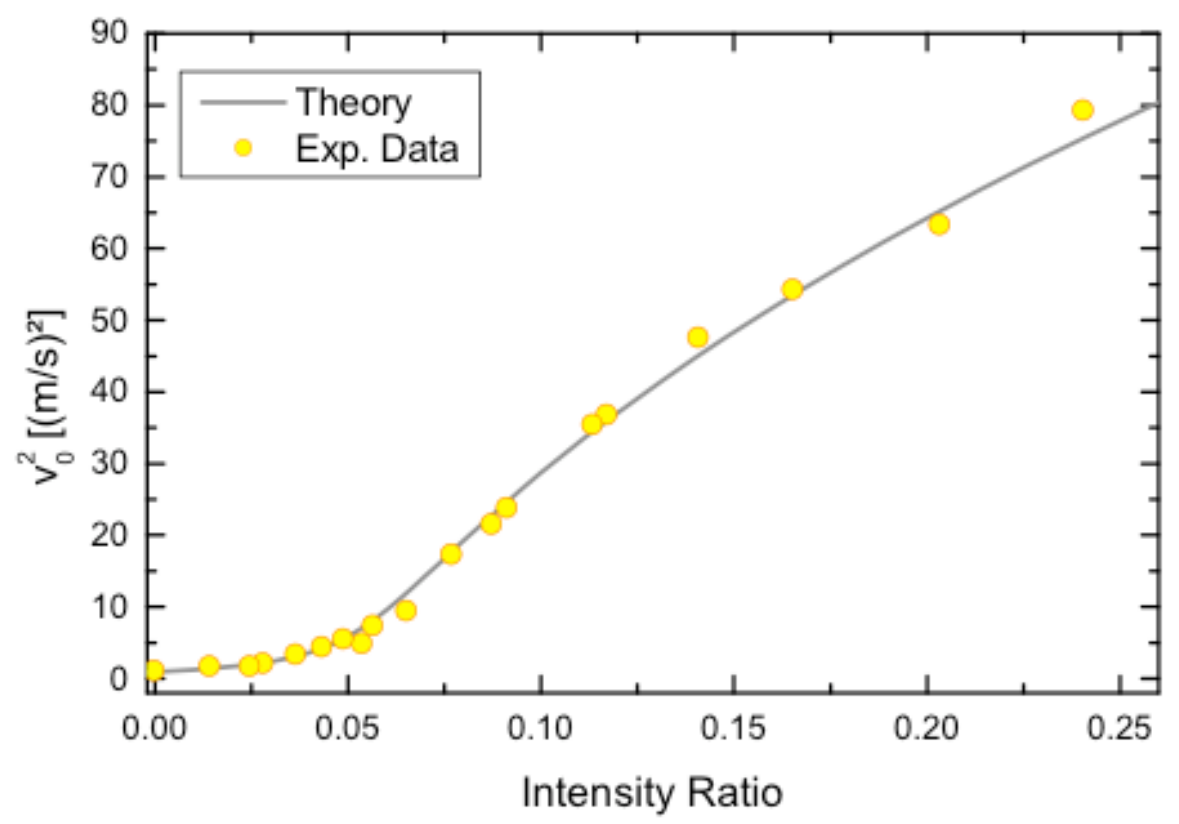

Figure 2: Measured velocity amplitude, $v_{0}^{2}$, plotted versus the intensity ratio $i_{a} / i_{c}$ for the magnesium ion system with cooling intensity $\left(\mathrm{i}_{\mathrm{c}}\right.$ ) held constant. The cooling and amplification beams are detuned by $-74 \mathrm{MHz}$ and $+12 \mathrm{MHz}$, respectively. The solid curve is a theoretical curve. There are no free parameters in the calculation.

\section{References}

[1] T.W. Hänsch and A.L.Schawlow, Opt. Communications 13 (1975).

[2] D.Wineland and H. Dehmelt, Bulletin American Physical Society 20 (1975).

[3] H.J. Kimble, Nature, 453, 1023 (2008).

[4] R. Blatt and D. Wineland, Nature, 453, 1008 (2008).

[5] K. Helmerson and W.D. Phillips, Rivista del Nuovo Cimento, 31, 141 (2008).

[6] I. Bloch, Science, 319, 1202 (2008).

[7] M. Herrmann, V. Batteiger, S Knünz, G. Saathoff, Th. Udem, and T. W. Hänsch, Physical Review Letters (in press). 